\title{
Trabajo con solución de problemas matemáticos en tercer grado de primaria: análisis en dos escuelas privadas
}

\author{
Yolanda Rosas Rivera ${ }^{1}$ \\ Yulia Solovieva ${ }^{2}$
}

\section{RESUMEN:}

El objetivo del presente artículo consiste en conocer y analizar la forma de trabajo de la solución de problemas que utilizan dos maestras con diferentes programas educativos (programa competencias-oficial y teoría de la actividad aplicada a la enseñanza). Se propone el análisis del método de enseñanza de la solución de problemas desde las aportaciones metodológicas de la teoría de la actividad. El método de investigación fue cualitativo. Como participantes se incluyeron a dos maestras de tercer grado y a sus alumnos. Se siguieron las siguientes fases: diseño de instrumentos, observación de clase, entrevista a maestras y evaluación cualitativa de alumnos. Los resultados muestran que un colegio forma habilidades operativas en los alumnos, mientras que otro colegio desarrolla habilidades generales para la solución de problemas. Concluimos que el conocimiento de la estructura de la solución de problemas favorece que los alumnos desarrollen habilidades generales y resuelvan los problemas de forma reflexiva.

PALABRAS CLAVE: solución de problemas, educación básica, teoría de la actividad, enseñanza de las matemáticas.

\section{Work with solving mathematical problems in third grade of primary school: analysis in two private schools}

\footnotetext{
${ }^{1}$ Doctorado en Educación Interinstitucional. Universidad Iberoamericana Puebla, Puebla, México. E-mail: npyolandarosas@gmail.com

${ }^{2}$ Doctorado en Psicología. Universidad Autónoma de Puebla, Puebla, México. E-mail: yulia.solovieva@correo.buap.mx
} 


\begin{abstract}
:
The objective of this article was to identify and analyze the way of teaching the solution of problems used by teachers within different educational programs (official-competency program and theory of the activity applied to teaching). The authors propose the way for analysis of teaching method for problem solving. This analysis is based on methodological contributions of activity theory. The method of research was qualitative. Participants of the study were teachers and pupils of the third grade. The phases of the study were as follows: design of the instruments of assessment, observation of working in classroom, interviews with teachers and qualitative evaluation of pupils. The results show that one school teach operational mechanic skills, while another school develops general skills for solving of mathematical problems. The authors conclude that the knowledge of the structure of the solution of problems allows development of general abilities in mathematic field together with reflexive thinking.
\end{abstract}

KEYWORDS: problem solving, activity theory, elementary school, mathematic teaching.

\title{
Introducción
}

La enseñanza de las matemáticas es una de las principales preocupaciones de la educación en México. Lo anterior se debe no solo por los resultados desfavorables que los alumnos de educación básica obtienen en pruebas nacionales (Planea, 2015) o internacionales (TERCE, 2015), sino también a la falta de claridad del contenido matemático y de su abordaje en las aulas. Desde el análisis de los programas de estudio para los maestros (SEP, 2011) es posible percibir la desarticulación que existe entre la teoría planteada con la metodología propuesta y la "realidad que se vive en las aulas". En dicho programa de estudio se refiere que "la perspectiva sociocultural o socioconstructivista de las competencias aboga por una concepción de competencia como prescripción abierta" (SEP, 2011) y enfatiza 
que el medio, por el cual se logran las competencias, es la inclusión de la solución de problemas particulares. Sucede que lo que está en el papel no coincide con lo que esta en la realidad, por lo cual las autores de este artículo coincidimos con la precisión que realiza Didriksson (2010) sobre la base de las competencias, la cual en esencia es un enfoque conductual y contradice toda visión sociocultural o socioconstructivista.

Dicha desarticulación se observa no solo a nivel teórico, sino también con la metodología planteada. Se enfatiza en "se hace al andar" (SEP, 2011), dejando los medios y procesos de enseñanza-aprendizaje de forma abierta. El maestro debe buscar la forma de evitar la enseñanza tradicional, con la cual desafortunadamente ha sido formado. Esto es posible percibirlo en la investigación de Arévalo (2015), quien se encargó de identificar las creencias de los maestros sobre el programa de estudio SEP 2011. De acuerdo con sus resultados los maestros opinan: "yo enseño con la misma metodología que me enseñaron... tradicional" "trato de buscar la manera más sencilla de explicarlo, pero aún así no todos me entienden que tienen que hacer". Además, en esta investigación se concluye que los maestros frecuentemente continúan transmitiendo información y pocas veces dejan espacios para que los alumnos participen.

Otro dato alarmante es que los maestros no solo se están enfrentando a un programa educativo que carece de una visión teórica y metodológica, sino que requieren del conocimiento propio de las matemáticas. Es decir, los conceptos, acciones y habilidades matemáticas que son necesarias formar en la educación básica no son claros para los maestros (ROSAS, SOLOVIEVA y QUINTANAR, 2017; ZARRAGA, SOLOVIEVA y QUINTANAR, 2017). De acuerdo con Ávila (2011), la solución de problemas ha sido un contenido relevante que surgió del cambio de una matemática tradicional a una matemática moderna. Debido a lo anterior, en la matemática tradicional el objetivo era la solución de algoritmos, los cuales eran aprendidos por transmisión y repetición. Por el contrario, la matemática moderna surgió de la necesidad de hacer pensar a los alumnos y se centró en el razonamiento 
matemático para construir nuevos conocimientos. Con todo, Ávila (2011) enfatiza en que la solución de problemas se utiliza frecuentemente para evidenciar lo que los niños pueden realizar, sin lograr realmente el objetivo de formar nuevos conocimientos.

Del análisis anterior del programa de enseñanza-SEP (basado en competencias) y la importancia de la solución de problemas como actividad intelectual surge nuestro objetivo de identificar cómo se enseña la solución de problemas en tercer grado de primaria y qué resultados se obtienen con esa enseñanza. Desde esta perspectiva, no sería pertinente enfocarse en un análisis aislado solo de uno de los agentes del proceso de enseñanzaaprendizaje, es decir, conocer solo las prácticas docentes o solo las habilidades que tiene los alumnos, sino conocer ese proceso de enseñanzaaprendizaje que surge en las aulas como un proceso integral (PUENTES, 2017).

Para lograr este objetivo, consideramos como primera necesidad seleccionar una teoría que permita analizar esa forma de enseñanzaaprendizaje y que oriente hacía los elementos esenciales tanto de las matemáticas como de la actividad escolar. Como segundo paso, decidimos usar la observación de clase, la entrevista al docente y una evaluación dinámica con los alumnos como elementos metodológicos.

En un primer momento expondremos la selección de la teoría que realizamos y en un segundo momento el trabajo de campo realizado.

\section{La teoría de la actividad aplicada a la enseñanza de las matemáticas}

Las autoras de este artículo han elegido la teoría de la actividad aplicada a la enseñanza porque, a pesar de no ser identificada en el área de educación, diversos autores han logrado organizar métodos de enseñanza novedosos y con resultados positivos en el desarrollo de la actividad intelectual (SOLOVIEVA, ROSAS y QUINTANAR, 2017; MORETTI y CEDRO, 2017; TALIZINA, 2017; SALMINA, 2017). La teoría de la actividad 
aplicada a la enseñanza ha sido desarrollada por los seguidores de Vygotski (TALIZINA, SOLOVIVEA y QUINTANAR, 2010; DAVIDOV, 1988; GALPERIN, 2009). Estos autores reconocen la cultura general humana como fuente del desarrollo psicológico de los alumnos (TALIZINA, 2009; VYGOTSKI, 1995). La actividad de la enseñanza y aprendizaje escolar se llevan a cabo desde una perspectiva cultural única que debe tomar en cuenta los contenidos y la forma de la interacción con ellos. Tanto los contenidos como la forma de interacción con ellos pueden ser analizados y organizados previamente, lo cual requiere de una reflexión teórica y metodológica de los participantes adultos en este proceso. Se puede decir que sólo la actividad de enseñanza que es organizada y dirigida favorece el aprendizaje y a el desarrollo de la actividad intelectual de los alumnos (SOLOVIEVA, 2004). Para que el maestro logre dicha organización y dirección es necesario que identifique el contenido esencial de los conceptos o habilidades matemáticas que formará en los alumnos, así como las acciones.

Desde el punto de vista de la organización reflexiva de la actividad, Nicola y Talizina (2017) consideran la solución de problemas como una actividad que requiere no solo el análisis de las matemáticas, sino también de la formación de habilidades generales del pensamiento. La solución de problemas inicia por determinar qué acciones se van a realizar por parte de los alumnos de forma reflexiva que requiere conocer y asimilar dichas acciones, no solo responder de forma caótica.

Una de las primeras acciones necesarias durante la solución de problemas es la de identificar de forma reflexiva la pregunta del problemas y de las condiciones, ante las cuales dicha pregunta se establece. Las condiciones del problema siempre describen una u otra situación, detrás de las cuales el alumno debe descubrir las relaciones aritméticas determinadas, es decir, debe describir la situación que se menciona en el idioma de las matemáticas. El maestro no simplemente debe presentar y anunciar el problema, sino mostrar qué hay que hacer para qué el alumno logre solucionar un problema. Para ello, se debe conocer de qué tipo de acciones 
intelectuales consiste el proceso de solución de cualquier problema de un grado escolar determinado y en qué orden se deben realizar (TALIZINA, 2009, 2017).

Cualquier problema aritmético incluye una pregunta final, cuya respuesta constituye el objetivo de la solución del problema. Para poder responder la pregunta final (o preguntas intermedias que llevan a la pregunta final) del problema, se tiene que analizar determinados datos, es decir, obtener la información necesaria y suficiente de los nexos matemáticos y lógicos formulados en el problema. En el contexto de un problema, dicha información se encuentra oculta detrás de los datos descriptivos del texto verbal. A partir de esta descripción verbal se tiene que obtener la informacion matemáticametne significativa y rechazar la información matemáticamete irrelevante. Lo anterior se logra solo mediante la actividad orientativo-investigativa, que constituye uno de los componentes de la actividad intelectual (TSVETKOVA, 1999).

Sólo sobre la base de los datos, obtenidos a partir de la actividad orientativo-investigativa, el alumno puede elaborar el esquema general para el contenido del problema estableciendo las relaciones esenciales entre los datos que se mencionada de la fomra directa e indirecta y las posibilidades que se tienen para responder a la pregunta final. Sólo después de esto, es factible encontrar las operaciones aritméticas que correspondan con el plan creado (LURIA y TSVETKOVA, 1981). En resumen los componentes de la estructura general de la solución de problemas son los siguientes: base orientadora de la acción, que planifica las actividades, las fases de la aplicación del sistema de operaciones concretas y finalmente del control de las acciones, lo cual permite comparar la respuesta con la pregunta del problema (TSVETKOVA, 1999).

Hasta aquí hemos presentado el marco teórico que nos orientó para realizar el análisis del contenido y estructura de la solución de problemas de matemáticos. A diferencia de otros enfoques (constructivista $\mathrm{y}$ competencias), la teoría de la actividad nos brinda la posibilidad de 
comprender el proceso de la solución de problemas, nos permite identificar las acciones propias y saber cuál requiere de mayor intervención.

Como siguiente paso para cumplir nuestro objetivo de conocer la forma de enseñanza de la solución de problemas fue necesario organizar la estructura análisis de la forma de trabajo con problemas. Es decir, era necesario determinar qué elementos se iban a observar en las clases, cuáles se iban a preguntar con los maestros y qué habilidades se iban a valorar con los alumnos. Para resolver esto decidimos considerar los elementos que son incluidos en los programas formativos que utilizaron Solovieva, Rosas y Quintanar (2017), Talizina (2009, 2017), Salmina (2017) y Nikola y Talizina (2017).

Estos autores identificaron los conceptos y habilidades matemáticas, las acciones necesarias para la formación de dichos conceptos, la orientación completa-generalizada e independiente que llevó a los alumnos a asimilar exitosamente. Siguiendo a ellos, nosotros proponemos analizar la forma de enseñanza-aprendizaje de las matemáticas mediante el método de enseñanza. Nosotros consideramos un método de enseñanza como la forma de organización y sistematización de los elementos necesarios para la enseñanza de una asignatura. Los elementos del método de enseñanza que hemos identificado son: 1) Sistema de conceptos o habilidades matemáticas. En el caso de las matemáticas, se puede identificar el concepto de número y sistema numérico como conceptos básicos y necesarios para lograr la solución de problemas matemáticos. 2) Tipo de acciones. En el caso de trabajo con problemas se trata de identificación del nivel de las acciones propias para los alumnos que puedan favorecer al proceso de una solución exitosa (GALPERIN, 2009; TALIZINA, 2009; SOLOVIEVA, 2004). 3) Tipo de orientaciones. Para el trabajo de problemas matemáticos se trata de orientación para identificación de las operaciones matemáticos detrás del contenido discursivo verbal del texto del problema (GALPERIN, 2009). 4) Formas de interacción. Se refiere a la forma de trabajo que predomina en el proceso concreto de enseñanza-aprendizaje escolar. Se 
puede tratar de trabajo en el grupo o individual de cada alumno, así como la interacción de la maestra con los alumnos. 5) Nivel de adquisición de conceptos matemáticos. En nuestro caso, esta actividad se refiere a la posibilidad de comprender y solucionar adecuadamente los problemas matemáticos.

De esta forma, analizamos el trabajo de solución de problemas en diferentes colegios dentro de la concepción global del proceso de enseñanzaaprendizaje y no de forma aislada. Este análisis incluye la realización de trabajo con los alumnos de una forma dinámica y no solo para identificar lo que ellos pueden realizar por sí solos y de forma empírica.

\section{Método}

Esta investigación se basó en una perspectiva cualitativa, debido al interés de conocer la forma de trabajo con la solución de problemas que existen en dos colegios con diferentes programas y su relación con las habilidades que desarrollan los alumnos. La forma en la que el investigador se inserta en el campo, a partir del método cualitativo, permite la interacción activa y flexible con el objeto de estudio y con los participantes, así mismo favorece el establecimiento de una constante comunicación para recolectar la información con diversos instrumentos (GONZÁLEZ, 2001).

\section{Participantes:}

La elección de los colegios fue de forma intencional y por conveniencia. Se escogieron dos colegios privados que declaraban por ellos mismo un método de enseñanza con resultados de acuerdo a lo que su programa de educación establecía.

En esta investigación se trabajó con dos maestras y sus alumnos correspondientes de tres grupos de tercer grado de primaria, de los cuales basan su enseñanza en: 1) la teoría de la actividad aplicada a la enseñanza (colegio Kepler) y 2) programa oficial (competencias). En total se trabajó con: 7 alumnos (colegio Kepler) y 9 alumnos (colegio con programa oficial). El 
grupo del colegio Kepler fue natural, mientras que en el colegio con programa oficial fueron elegidos por tener un mejor rendimiento académico en las matemáticas. El colegios Kepler se encuntra en la ciudad de Puebla, mientras que el colegio oficial se ubica en la ciudad de México. Los dos colegios pertenecen a sectores sociales urbanos.

En la siguiente tabla se caracterizan a los participantes:

TABLA 1. Características de las maestras

\begin{tabular}{|c|c|c|c|c|}
\hline Maestra & Edad & Formación & $\begin{array}{c}\text { Años de } \\
\text { docencia }\end{array}$ & $\begin{array}{c}\text { Cursos de } \\
\text { profesionalización }\end{array}$ \\
\hline Oficial & 43 & $\begin{array}{c}\text { Licenciatura en } \\
\text { educación }\end{array}$ & 6 años & $\begin{array}{c}\text { CONOCER } \\
\text { (modelo centrado en } \\
\text { competencias) }\end{array}$ \\
\hline Kepler & 41 & $\begin{array}{c}\text { Licenciatura en } \\
\text { educación }\end{array}$ & 13 años & $\begin{array}{c}\text { Dibujo, cuento, concepto } \\
\text { de número }\end{array}$ \\
\hline
\end{tabular}

Fuente: Archivo de las autoras.

Fue posible identificar que las maestras se encuentran en capacitación constante, proporcionada en sus respectivos colegios. Ambas maestras han trabajado la mayor parte en colegios privados con los grados de tercero de primaria.

A continuación se presentan las características de los alumnos, especificando el colegio.

TABLA 2. Características de los alumnos

\begin{tabular}{|l|c|c|c|c|c|}
\hline \multirow{2}{*}{ Colegio } & \multicolumn{3}{|c|}{ Alumnos } & Edad & Años cursados \\
\cline { 2 - 4 } & Total & Niñas & Niños & promedio & en el colegio \\
\hline Kepler & 7 & 1 & 6 & 8.8 & 4 \\
\hline Oficial & 9 & 6 & 3 & 8.5 & 3.8 \\
\hline
\end{tabular}

Fuente: Archivo de las autoras.

\section{Instrumentos:}


Los instrumentos que se utilizaron fueron una entrevista semiestructurada, un protocolo de evaluación de conceptos matemáticos para alumnos y la hoja de registro de la observación de clases. Los instrumentos fueron diseñados a partir de la teoría de la actividad y de las investigaciones en el área de la enseñanza de las matemáticas desde el mismo enfoque. La estrategia de análisis de los datos obtenidos a partir de los instrumentos fue de contenido (FLICK, 2007; MAYARING, 2000).

1) La entrevista semi-estructurada para las maestras fue diseñada a partir de constructos teóricos.

2) Protocolo de evaluación de la solución de problemas matemáticas. La evaluación se realizó a través de un proceso interventivo (dinámico) propuesto por Vygotsky y sus seguidores (VYGOTSKY, 1995; TALIZINA, SOLOVIEVA y QUINTANAR, 2010).

3) Hoja de registro de observación de clases. Esta fue organizada para identificar los elementos del método de enseñanza durante las clases: tipo de orientación, acciones que incluye, plano de ejecución de las actividades, evaluación de las tareas, organización de las participaciones, tipos de ayuda.

\section{Procedimiento:}

A partir del tipo de investigación fueron organizadas las siguientes fases: 1) diseño de instrumentos, 2) elección intencional de participantes, 3) observación no participativa en clases (tres clases con duración de una hora), 4) aplicación de la entrevista semi-estructurada a maestras, 5) evaluación dinámica con los alumnos (forma individual, con una duración de 40 minutos).

\section{Resultados}

De forma general fue posible identificar las siguientes características del método de enseñanza que utilizan las maestras para trabajar con la solución de problemas. Primero describiremos la forma en que las maestras 
trabajan la solución de problemas en el aula, y posteriormente describiremos los resultados de la evaluación realizada a los alumnos.

\section{Método de enseñanza de la maestra del colegio oficial}

En el colegio oficial, la maestra refiere que la solución de problemas es una tarea importante, específicamente en tercero de primaria, ella trabaja con problemas que implican dos operaciones. La dificultad que la maestra observa en esta actividad es que los alumnos no comprenden el problema, para lo cual realiza lectura con los alumnos y les enfatiza que en el mismo problema se dice lo que deben hacer para solucionar. Además, ella utiliza como estrategia la identificación de palabras clave, por ejemplo, si el problema dice "regalaron", entonces implica una suma, si dice "perdieron", entonces es resta, mientras que "repartieron" es división. A partir de la observación en clase fue posible identificar algunas características propias de los procedimientos que se utilizan.

El problema que se trabajó en el aula fue: "Ramiro tiene un costal de 157 paquetes de cacahuates y los va repartiendo en 12 paquetes, ¿cuántos paquetes tiene cada uno? ¿Le sobran? ¿Cuántos?”

La maestra lo soluciona con sus alumnos, ella escribe en el pizarrón y pasa a los alumnos a resolver la operación. La maestra escribe en el pizarrón: datos, operación y resultados. Después interactúa con los alumnos de la siguiente forma:

Maestra: dime (mirando a los alumnos) ¿cuáles son los datos? ¿De qué está hablando el problema? ¿Lo números qué?- Un alumno: "paquetes de cacahuates" - Maestra: paquetes de cacahuates, ¿cuántos? Dime el número, y luego, entre doce, aquí lo voy a poner yo espero que ustedes le vayan entendiendo, entonces vamos a ver la operación por supuesto que es...- Una alumna: 30 - Maestra: no, la operación -Otra alumna: 157 entre 22, -Maestra: exacto, ¿cómo hicieron esto?, ahorita si yo ya no les pedí es como ustedes quisiera 
con resta o sin resta (algoritmo de división por resta o uso de multiplicación).

Después pide a una alumna pasar al pizarrón y resolver la operación.

A partir de la descripción anterior es posible percibir que la orientación que la maestra utiliza es incompleta, el contenido de la tarea no es suficiente para resolver correctamente el problema, se dirige hacia la solución de un algoritmo sin comprender completamente esta necesidad. La forma de organización de la tarea es operativa con una reflexión sobre los datos matemáticos y los datos conductuales (pregunta-respuesta). Podemos considerar que la maestra da mayor importancia a la identificación de datos y a la operación. La primera pregunta que realiza para el análisis del problema es “dime, ¿cuáles son los datos?” y posteriormente “¿de qué habla el problema?" a lo que una alumna responde sobre "paquetes de cacahuates", la maestra repite la pregunta “¿cuántos?, dime el número” y escribe estos datos en el pizarrón, sin analizar esa situación en la que participan los números. Lo que sigue es identificar la operación, para lo cual la maestra enfatiza la palabra "entre" y considera que esto es necesario para que los alumnos asocien con una división. Por último, las acciones que utiliza la maestra son solo en plano verbal externo, los alumnos usan solo la escritura.

\section{Método de enseñanza de la maestra del colegio Kepler}

Durante la entrevista, la maestra del colegio Kepler mencionó que la solución de problemas "tiene como objetivo el razonamiento e implica dar una respuesta a una pregunta, un análisis de datos, así mismo no todos los problemas tienen solución, hay datos que sobran, que son tramposos". Para esta última actividad la maestra menciona que utiliza problemas (escritos o dictados) con situaciones cotidianas, en ocasiones los alumnos inventan, dan valores y diseñan sus propios problemas. Las dificultades que la maestra observa en sus alumnos son la identificación de la operación para lo cual 
considera que la tarea de que ellos diseñen sus problemas les facilita su aprendizaje.

Durante la observación de clase fue posible identificar la siguiente dinámica: la maestra dicta y escribe en el pizarrón el problema, espera a que los alumnos escriban. El problema fue el siguiente: Isa, tiene una caja con 2556 manzanas, cinco novenos son rojas, y el resto verdes, ¿cuántas manzanas rojas hay? ¿Cuántas manzanas verdes hay? ¿Cuántas manzanas hay?.

La maestra les recuerda que van a trabajar con su tarjeta de orientación y les reparte a cada alumno, para que la tengan en sus manos durante el trabajo. Posteriormente, la maestra solicita que "alguien nos ayude a leer la tarjeta de solución de problemas" y un alumno participa y lee la tarjeta de orientación: “a) lea el problema, b) con tus palabras menciona qué es lo que se pregunta en el problema, c) escribe que datos conoces, d) responde si con los datos que tienes, puedes responder la pregunta, e) elige la operación matemática necesaria y escribe la formula, f) realiza los pasos necesarios para resolver la operación, g) verifica el resultado, este resultado debe responder a la pregunta final del problema.

Posteriormente, la maestra enfatiza en esos pasos, explicando cada uno a los alumnos: "vamos a trabajar en equipos para poder resolver, cuando tengan el trabajo lo vamos verificando en el pizarrón... de acuerdo... lo vamos haciendo...datos, formula, operación y resultado, y después lo que trabajamos es nuestro esquema y lo representamos: a) primero tenemos que leer nuestro problema, b) mencionar que es lo que falta para contestar a la pregunta del problema, c) escribir qué datos conocemos, acuérdense de que hay problemas donde sobran datos, faltan y donde están lo que se necesita, acuérdense que hay problemas que no se pueden resolver (faltan datos), e) después tenemos que responder ¿con los datos, de los que tenemos, podemos responder la pregunta?, f) después elegir qué operación vamos a utilizar para resolver el problema, y g) bueno... ya después a seguir los pasos 
necesarios, vamos viendo nuestra incógnita, y ya con nuestra operación damos el resultado a la pregunta del problema".

Es posible identificar que en todo el procedimiento y la interacción con los alumnos, la maestra muestra un estructura de la solución de problemas, la cual inicia con la identificación de la pregunta final del problema. Además, la maestra utiliza la reflexión para identificar los datos y su relación, no solo de forma matemática sino también de forma lógica, cuando comenta que las características de los datos (datos necesarios, faltantes, insuficientes).

Durante el trabajo, la maestra utiliza las acciones en el plano materializado, porque les brinda a los alumnos pedazos de hojas para representar las medidas de fracciones. Posteriormente ellos tienen que representarlo mediante su esquema y resolver en plano verbal externo (escritura). También es posible observar que la maestra utiliza la interacción alumno-objeto cuando les solicita materializar la medida; también utiliza la interacción alumno-alumno, debido a que los problemas los resolvían en parejas y después de forma grupal. Por último fue posible percibir la interacción maestra-grupo. En la siguiente tabla (4) se resumen las características del método que utiliza cada maestra.

TABLA 4. Métodos de enseñanza de la solución de problemas

\begin{tabular}{|c|c|c|c|c|}
\hline Colegio & $\begin{array}{c}\text { Solución de } \\
\text { problemas }\end{array}$ & Orientación & $\begin{array}{c}\text { Tipo de } \\
\text { acciones }\end{array}$ & $\begin{array}{c}\text { Tipo de } \\
\text { colaboración }\end{array}$ \\
\hline Oficial & $\begin{array}{l}\text { Habilidades } \\
\text { operativas } \\
\text { para la } \\
\text { solución de } \\
\text { problemas }\end{array}$ & $\begin{array}{l}\text { Incompleta, } \\
\text { particular y } \\
\text { dependiente } \\
\text { (maestra) }\end{array}$ & $\begin{array}{l}\text { Acciones } \\
\text { verbales } \\
\text { externas } \\
\text { (escritura de } \\
\text { algoritmos) }\end{array}$ & $\begin{array}{l}\text { Alumno- } \\
\text { maestro }\end{array}$ \\
\hline Kepler & $\begin{array}{l}\text { Habilidades } \\
\text { generales } \\
\text { para la } \\
\text { solución de } \\
\text { problemas }\end{array}$ & $\begin{array}{l}\text { Completa, } \\
\text { generalizada e } \\
\text { independiente }\end{array}$ & $\begin{array}{c}\text { Acciones } \\
\text { materializadas, } \\
\text { perceptivas y } \\
\text { verbales } \\
\text { externas }\end{array}$ & $\begin{array}{l}\text {-Alumno- } \\
\text { objeto } \\
\text {-Alumno- } \\
\text { alumno } \\
\text {-Alumno- } \\
\text { maestro }\end{array}$ \\
\hline
\end{tabular}

Fuente: Archivo de las autoras. 


\section{Evaluación de alumnos.}

Una vez descritos los elementos del método de enseñanza de la solución de problemas que identificamos en cada colegio, pasaremos a los resultados de la evaluación dinámica aplicada a los alumnos. La evaluación se aplicó de manera individual, tuvo una duración de 40 minutos. Los alumnos eran retirados de actividades que no afectaban su aprendizaje o interés de otras asignaturas. Los alumnos del colegio Kepler y oficial fueron retirados uno a uno de su salón y se trabajó con ellos en otro salón.

De forma general, todos los alumnos aceptaron participar en la investigación, ellos mostraron intéres en las tareas y aceptaban los tipos de apoyo de forma positiva. Además, la mayoría de los alumnos del Kepler refirió gustarles mucho las matemáticas, mientras que la mayoría del colegio oficial mencionaron que no les gustaba porque eran difíciles. No obstante, todos los alumnos mostraron agrado por la forma en la que les enseñan sus maestras.

En la siguiente tabla (5) se muestran los tipos de apoyo y errores que necesitaron los alumnos para resolver los problemas. En total los alumnos resolvieron 11 problemas. Los tipos de problemas planteados incluyeron las cuatro operaciones matemáticas, y se pueden organizar como: 1) simples, que implican una operación matemática, con variante en situación cotidiana (libros/estantes) y no cotidiana $(\mathrm{km} / \mathrm{h})$, ejemplo: Un tren avanzó $98 \mathrm{~km}$ durante 8 horas. ¿Cuántos kilómetros hace el tren en una hora?., 2) problemas complejos, los cuales implican la solución de dos operaciones (conversión de medidas, inferir un dato), por ejemplo: Renata y Daniel fueron al mercado y compraron lo siguiente: 2 kilos de manzana, 300 gramos de azúcar y 1 kilo de pasta, ¿cuántos gramos compraron en total?, 3) problemas sin solución, los cuales tenían una situación y una pregunta final más estos no tenían relación, ejemplo: Durante 12 días se construyó una carretera de 48 kilómetros, ¿cuántos carros pasaron durante un día? 
En la siguiente tabla (5) se muestran los tipos de error y de apoyo que necesitaron los alumnos, es importante mencionar que en el Colegio Kepler solo un alumno tuvo respuestas incorrectas y requirió de los apoyos que se mencionaran, sin embargo este alumno cuenta con un diagnóstico neurológico.

TABLA 5. Resultados de alumnos en problemas complejos

\begin{tabular}{|c|c|c|}
\hline Colegio & Oficial & Kepler \\
\hline $\begin{array}{l}\text { Tipo de } \\
\text { error }\end{array}$ & $\begin{array}{l}\text {-Identificar la medida. } \\
\text {-Valor jerárquico del numero. } \\
\text {-Organizar datos en el } \\
\text { algoritmo. }\end{array}$ & -Identificar la medida. \\
\hline $\begin{array}{l}\text { Tipos de } \\
\text { apoyo }\end{array}$ & $\begin{array}{l}\text {-Explicación del uso de la } \\
\text { medida. } \\
\text {-Escritura de la conversión y } \\
\text { organización de datos. }\end{array}$ & $\begin{array}{l}\text {-Escritura de la conversión y } \\
\text { organización de datos. }\end{array}$ \\
\hline
\end{tabular}

Fuente: Archivo de las autoras.

Los alumnos del colegio Kepler respondieron correctamente, identificaron la pregunta final y los datos con sus medidas, solo un alumno requirió de recordarle la conversión de kilogramos a gramos.

En la siguiente tabla (6) se muestran los tipos de errores y ayudas que solicitaron los alumnos en problemas simples.

En el tipo de problemas anterior, la mayoría de los alumnos del colegio oficial resolvían de forma automatizada, verbal externa y con ayuda de sus dedos. Ellos identificaban los datos y realizaban la operación. Sin embargo, estos alumnos no lograban explicar sus resultados, solo verificaban matemáticamente, es decir, mencionaban "la respuesta es $21 \ldots$ porque $7 \times 3$ es 21 ” además cometían muchos errores de conteo “7 x 3 es 20 , no 18 , no $23 \ldots 7 \times 1=7,7 \times 2=15,14,7 \times 3=21$ " para esto se les ayuda a identificar su medida en el plano perceptivo (dibujo de 7 palitos de conteo) y aumentarla tres veces.

En la siguiente tabla (7) se muestran los tipos de errores y de apoyos que solicitaron los alumnos para resolver problemas que no tenían solución. 
TABLA 6. Resultados de alumnos en problemas simples

\begin{tabular}{|c|c|c|}
\hline Colegio & Oficial & Kepler \\
\hline $\begin{array}{l}\text { Tipo de } \\
\text { error }\end{array}$ & $\begin{array}{c}\text {-Comprensión de la relación } \\
\text { entre los datos } \\
\text {-Dificultad para atender } \\
\text { pregunta final } \\
\text {-Conteo estimación } \\
\text {-Organización de los datos en el } \\
\text { algoritmo } \\
\text {-Verificación de resultados }\end{array}$ & $\begin{array}{l}\text {-Explicar su resultado } \\
\text { (reflexión de su resultado) }\end{array}$ \\
\hline $\begin{array}{l}\text { Tipos de } \\
\text { ароуо }\end{array}$ & $\begin{array}{c}\text {-Dibujo de un esquema para } \\
\text { explicar la relación entre los } \\
\text { datos } \\
\text {-Repetición del problema } \\
\text {-Explicación de la relación } \\
\text { lógico-matemática de los datos } \\
\text {-Organización del algoritmo de } \\
\text { forma escrita }\end{array}$ & $\begin{array}{c}\text {-Dibujo de un esquema para } \\
\text { explicar la relación entre los } \\
\text { datos } \\
\text {-Repetición del problema }\end{array}$ \\
\hline
\end{tabular}

Fuente: Archivo de las autoras

TABLA 7. Resultados de alumnos en problemas sin solución

\begin{tabular}{|c|l|}
\hline Colegio & \multicolumn{1}{c|}{ Oficial } \\
\hline $\begin{array}{c}\text { Tipo de } \\
\text { error }\end{array}$ & $\begin{array}{l}\text {-Comprensión de la relación entre los datos } \\
\text {-Comprensión de la pregunta final } \\
\text {-Comprensión de la relación entre la situación y pregunta del } \\
\text { problema }\end{array}$ \\
\hline $\begin{array}{c}\text { Tipos de } \\
\text { apoyo }\end{array}$ & $\begin{array}{l}\text {-Repetición del problema } \\
\text {-Explicación y preguntas dirigidas hacia el análisis del } \\
\text { datos, selección de operación, respuesta a la pregunta del } \\
\text { problema) }\end{array}$ \\
\hline
\end{tabular}

Fuente: Archivo de las autoras

En este tipo de problemas, los alumnos del colegio oficial tuvieron dificultades para comprender el problema. Por ejemplo, este tipo de problemas fue "Durante 12 días se construyó una carretera de 48 kilómetros, ¿cuántos carros pasaron durante un día?” Las respuestas de los alumnos del colegio Oficial fueron 48/12, 48/1,12+48, fue posible observar que estos alumnos desde el inicio proponían la operación y cuando se les 
pregunta por qué entonces se detenían y al no lograr responder (algunos se quedaban callados otros decían que era una división) accedían al tipo de ayuda del evaluador. No obstante, los alumnos del colegio Kepler respondieron correctamente "no tiene solución" "cómo voy a saber cuántos carros sino me dice" "es un problema con trampa".

\section{Discusión}

A partir de nuestro objetivo que consistió en conocer la forma de enseñanza de la solución de problemas matemáticos en tercer grado de primaria de dos colegios con diferentes programas educativos, fue identificado a partir de la propuesta de nuestra categoría de análisis "método de enseñanza" desde la teoría de la actividad. Los métodos de enseñanza que identificamos fueron: en el colegio oficial fue posible percibir el trabajo con habilidades particulares-operativas, los alumnos tienen como objetivo solucionar una operación matemática, a partir de la estimación de resultados. Debido a lo anterior, la solución de problemas en este colegio se simplifica a su elemento operativo y desorganizado (TVSTEKOVA, 1999; TALIZINA, 2017; SALMINA, 2017).

El colegio Kepler, al tener una estructura de la solución de problemas que es dada en una tarjeta, se garantiza que los alumnos formen las habilidades generales y no solo operativos. Los alumnos saben en qué elementos deben orientarse (pregunta, datos, relación entre datos y respuesta a la pregunta) y también conocen otros tipos de problemas, no solo los que tienen solución directa o refieren una situación perceptiva directa (TALIZINA, 2009). Esta estructura, aunque la maestra no la declara de forma directa, es la que favorece el desarrollo de la actividad intelectual de acuerdo con Tsvetkova (1999) y Solovieva (2004).

Los resultados que obtienen las maestras a partir de su enseñanza fueron identificados con las tareas propuestas. Los alumnos del colegio oficial requirieron mayor apoyo de explicación de la estructura de la solución de problemas, es decir, explicarles qué es un problema matemático, en qué 
consiste (situación problemática, pregunta final), la relación que puede existir entre los datos se declara en la pregunta final y la respuesta debe ser hacia la pregunta final, no solo de la operación. También fue posible percibir que los alumnos del colegio Oficial han generado una dependencia de su maestra, los alumnos requieren demasiado acompañamiento del adulto para solucionar correctamente las tareas.

En el colegio Kepler, al hacer uso de tarjetas de orientación (pasos de análisis de los problemas matemáticos) genera que los alumnos puedan trabajar de forma independiente, fue posible observar que algunos alumnos no necesitaban su tarjeta, ya conocían los pasos y realizaban, así mismo cuando tenían dudas se orientaban en estos pasos. Este carácter independiente es importante para la asimilación de conceptos y de acciones, de acuerdo con Galperin (2009) y Talizina (2017), permite que los alumnos identifiquen el contenido esencial y resuelvan tareas de forma reflexiva. Sin embargo, desde nuestro punto de vista, la solución de problemas es vista solo como un ejercicio más que requiere de conocimiento previo de las matemáticas (SILVA y RODRÍGUEZ, 2011), más no se ha logrado enfatizar en los elementos que incluye. En los programas SEP (2011) se refiere que los alumnos deben solucionar problemas y crear sus propias estrategias, pero no muestra qué significa esto, hacia donde debe ser orientado y organizado el pensamiento de los alumnos.

Consideramos que esta falta de orientación psicológica es una de las limitaciones en las aulas escolares. Las maestras desconocen en qué consiste la solución de problemas y de qué condiciones depende el éxito de la solución. Los problemas se conciben como desafíos, los cuales están desorganizados, aislados y tienen una repuesta inmediata. Como consecuencia, los alumnos no logran comprender la relación que existe entre medidas que no son perceptibles por ellos, por ejemplo, la relación entre metros y horas.

En relación con las acciones que utilizan las maestras, también fue posible identificar que en el colegio Kepler se utiliza la formación de las 
acciones intelectuales por etapas. Para la solución de problemas la maestra utiliza las acciones materializadas, perceptivas y verbales externas (escritura). A diferencias de lo anterior, en el colegio Oficial existen solo acciones verbales (escritura y descripción), por lo que la repetición y solución de ejercicios aislados es lo que prevalece.

Respecto a las formas de comunicación en grupo, en el colegio Kepler se utiliza comunicación grupal que incluye a todos los participantes, mientras que en el colegio oficial predomina la comunicación dual entre maestro y uno de los alumnos sin lograr la inclusión de todo el grupo en la solución de un mismo problema.

\section{Conclusiones:}

La solución de problemas puede ser enseñada de forma orientativageneral o de forma operativa-concreta. Los alumnos logran explicar sus resultados cuando desarrollan habilidades generales, por el contrario, cuando solo se ocupan de identificar la operación concreta, dependen plenamente del apoyo concreto del maestro.

El uso de tarjetas de orientación, como se observó en el colegio Kepler, favorece la asimilación de los alumnos, no solo depender de las instrucciones verbales, sino plasmar el contenido esencial en tarjetas permite que los alumnos realicen las acciones necesarias y que conozcan los procesos que implican la solución de problemas.

Considerar a la solución de problemas como actividad intelectual exige conocimientos de una teoría psicológica, no solo de la didáctica ni de la educación matemática. Es posible percibir que las carencias y contradicciones en el programa oficial de la SEP son porque no existe con claridad una teoría psicológica. La maestra que utiliza este programa solo propone tareas aisladas, enfatiza la solución del algoritmo como principal objetivo de la solución de problemas.

La teoría de la actividad aplicada a la enseñanza permite la organización de la solución de problemas y su posible valoración en las 
aulas. Mediante nuestra propuesta de método de enseñanza y su contenido, los maestros podrían identificar cómo es su forma de enseñanza y proponer cambios que favorezcan el desarrollo de la actividad intelectual.

\section{Referencias Bibliográficas}

ÁVILA, A. En matemáticas ¿qué nos dejaron las reformas de fin de siglo XX?. Cuadernos de Investigación y Formación en Educación Matemática, Costa Rica, No. 9, 2011.

DAVIDOV, V. V. La enseñanza escolar y el desarrollo psíquico. Moscú: Progreso, 1988.

GALPERIN, P. Sobre la formación de las imágenes sensoriales y de los conceptos. En QUINTANAR, Luis; Solovieva, Yulia (Org.). Las funciones psicológicas en el desarrollo del niño. México: Trillas, 2009.

GONZÁLEZ, L. Investigación cualitativa en Psicología: Rumbos y desafíos. México: Paranhinfo, 2001.

MARTÍNEZ, G. El tránsito de la formación de conceptos matemáticos primarios a la solución de problemas aritméticos en niños de edad prescolar mayor y de edad escolar menor. Revista Cubana de Psicología, Cuba, v. 1 n. 1. (1984).

MORETTI, V. D., CEDRO, W. L. (Orgs.). Educação Matemática e Teoria HistóricoCultural: um olhar para a pesquisa. Mercado de Letras: Campinas, pp- 291-328, 2017.

NIKOLA, G.; TALIZINA, N. La formación de habilidades generales para la solución de problemas aritméticos. En TALIZINA, N.; SOLOVIEVA, Y.; QUINTANAR, L. Enseñanza de las matemáticas desde la teoría de la actividad. México: CEIDE, 2017.

LURIA, A.; TSVETKOVA, L. La resolución de problemas y sus trastornos. Barcelona: Editorial Fontanella, 1981.

ROSAS, Y. Análisis de los métodos de enseñanza de las matemáticas desde la teoría de la actividad. Tesis para obtener el grado de Doctor en Educación, Universidad Iberoamericana de Puebla, 2019 (en imprenta).

; SOLOVIEVA, Y.; QUINTANAR, L. La formación del concepto de número: aplicación de la metódica en una institución mexicana. En: TALIZINA, N.; SOLOVIEVA, Y.; QUINTANAR, L. Enseñanza de las matemáticas desde la teoría de la actividad. México: CEIDE, pp. 107-128, 2017.

SALMINA, N. La enseñanza de las matemáticas en la escuela primaria. En: TALIZINA, T.; SOLOVIEVA, Y.; QUINTANAR, L. Enseñanza de las matemáticas 
desde la teoría de la actividad. México: Centro de Investigación y Desarrollo educacional, pp. 23-68, 2017.

SILVA, M.; RODRÍGUEZ, A ¿Por qué fallan los alumnos al resolver problemas matemáticos? Didac, México, n. 56-57, pp. 21-28,2011.

SECRETARIA DE EDUCACIÓN PÚBLICA. Resultados nacionales 2015 de $6^{\circ}$ de primaria y $3^{\circ}$ de secundaria, Lenguaje y comunicación, Matemáticas. Recuperado en: http://www.inee.edu.mx/index.php/planea

tercer grado. México: SEP.

(2011). Programa de estudio 2011. Guía para el maestro. Primaria,

SOLOVIEVA, Y.; ROSAS, Y.; QUINTANAR, L. Programa para solução de problemas como método para desenvolvimento de pensamento lógico em crianças em idade escolar. En: MORETTI, V. D., CEDRO, W. L. (Orgs.). Educação Matemática e Teoria Histórico-Cultural: um olhar para a pesquisa. Mercado de Letras: Campinas, pp- 291-328, 2017.

Desarrollo del intelecto y su evaluación. Una aproximación históricocultural. México: Benemérita Universidad Autónoma de Puebla, 2004.

TALIZINA, N.; SOLOVIEVA, Y.; QUINTANAR, L. La aproximación de la actividad en psicología y su relación con el enfoque histórico-cultural de L. S. Vygotsky. Novedades educativas, n. 230, pp. 4-8, 2010.

La teoría de la actividad aplicada a la enseñanza. México: Benemerita Universidad Autónoma de Puebla, 2009.

La formación de los conceptos matemáticos. En: TALIZINA, N.; SOLOVIEVA, Y.; QUINTANAR, L. Enseñanza de las matemáticas desde la teoría de la actividad. México: CEIDE, pp.87-105, 2017.

TERCE. Aportes para la enseñanza Matemática. Chile: LLECE, 2016.

TSVETKOVA, S. Neuropsicología del intelecto. La Habana: UAEM, 1999.

PUENTES, R. V. Didática desenvolvimental da actividade: o sistema ElkoninDavidov (1958-2015), Obutchénie: Revista de Didática e Psicologia Pedagógica, Brasil, v. 1, n.1. 2017.

VYGOTSKI, L. Obras escogidas: Tomo 3. Madrid: Visor, 1995.

ZÁRRAGA, S.; SOLOVIEVA, Y.; QUINTANAR, L. Formación de las habilidades matemáticas básicas en preescolares mayores. En: TALIZINA, N.; SOLOVIEVA, Y.; QUINTANAR, L. Enseñanza de las matemáticas desde la teoría de la actividad. México: CEIDE, pp. 69-85, 2017. 